\title{
Model modulation to add smaller scale structures to large scale electron density models
}

\author{
R. Leitinger, E. Feichter, and M. Rieger \\ Institut für Geophysik, Astrophysik und Meteorologie, Universität Graz, Austria
}

\begin{abstract}
Usually regional and global electron density models provide large scale spatial structures only and smooth out the smaller scale features of the electron density distribution. We present a method to modulate existing electron density models by multiplication: $M(h, \varphi, \lambda, t)=L(h, \varphi, \lambda, t) \times$ $S_{1}(h, \varphi, \lambda, t) \times S_{2}(h, \varphi, \lambda, t) \times \cdots S_{n}(h, \varphi, \lambda, t)$

$M$ : resulting electron density distribution, $L$ : large scale model, $S_{1} \cdots S_{n}$ : modulating models for $\mathrm{n}$ the smaller scale structures; $h$ : height; $\varphi, \lambda$ : geographic coordinates, $t$ : Universal Time. There are no restrictions to the nature of the large scale model provided it takes height and horizontal coordinates as input. Examples are models of the "profiler" type which use large scale "maps" for profile anchor points (e.g., E, F1, F2 peak properties) like the International Reference Ionosphere (IRI). Typical examples for smaller scale structures are ridges, troughs and wavelike disturbances. The advantage of modulation by multiplication is that there is no danger to get zero or negative values of electron density as long as the background and modulations are $>0$ everywhere. For each modulation model, unity means "undisturbed".
\end{abstract}

\section{Introduction}

Large scale electron density models of the "profiler" type like the International Reference Ionosphere (IRI)(Bilitza, 2001) or the "model family" developed at Graz and Trieste use anchor points to which the height profile of electron density is attached. These anchor points are the peaks of the ionospheric "layers" E, F1 und F2. For monthly median conditions the models use "maps" from which the anchor point properties are derived. The "maps" are algorithms which allow to calculate the critical frequencies foE, foF1, foF 2 and the F2 layer transfer parameter M3000(F2).

The profiler models mentioned allow updating with measured values, a procedure adequate only for single height

Correspondence to: R. Leitinger

(reinhart.leitinger@uni-graz.at) profiles of electron density. Updating of slant profiles, e.g., along straight lines between arbitrarily chosen end points, could be done on a regional basis with special maps constructed by means of "instantaneous mapping procedures". For the model family NeQuick, COSTprof and NeUoGplas (Leitinger et al., 2000) a "data grid" method was proposed, successfully implemented and used (Leitinger et al., 2001, 2002). The global grids have a spacing of $2.5^{\circ}$ in latitude and $5.0^{\circ}$ in longitude. A cyclic third order interpolation scheme is used to gain values between the grid points. The latitude and longitude resolution of the data grids is comparable to that of the ITU-R (formerly CCIR) maps.

The map or data grid resolution is sufficient for large scale ionospheric structures only. Necessarily the temporal resolution is coarse too: the ITU-R maps exist for each month and each hour UT only. Data grids could be constructed for denser temporal spacing but it is not realistic to plan for time intervals smaller than $15 \mathrm{~min}$.

Our modulation method allows to superimpose smaller scale and time dependent structures.

\section{The modulation method}

Assuming that the large scale model is given as $L=L(h, \varphi, \lambda, t)$ and that we have several different modulations $\left(S_{1}, S_{2}, \cdots S_{n}\right)$ we gain the resulting electron density distribution $M$ as

$$
\begin{aligned}
M(h, \varphi, \lambda, t)= & L(h, \varphi, \lambda, t) \times S_{1}(h, \varphi, \lambda, t) \times \\
& S_{2}(h, \varphi, \lambda, t) \times \cdots S_{n}(h, \varphi, \lambda, t)
\end{aligned}
$$

$h$ : height; $\varphi, \lambda$ : geographic coordinates, $t$ : Universal Time.

$S_{1} \cdots S_{n}$ must be positive definite functions (being everywhere and at all times $>0)$, unity $\left(S_{k}=1\right)$ meaning "no disturbance", $0<S_{k}<1$ meaning "electron density depression", $S_{k}>1$ meaning "electron density enhancement".

The use of time dependent modulations necessarily needs time dependent large scale models. This can be realized by interpolation of the data grids. If the application interval of 


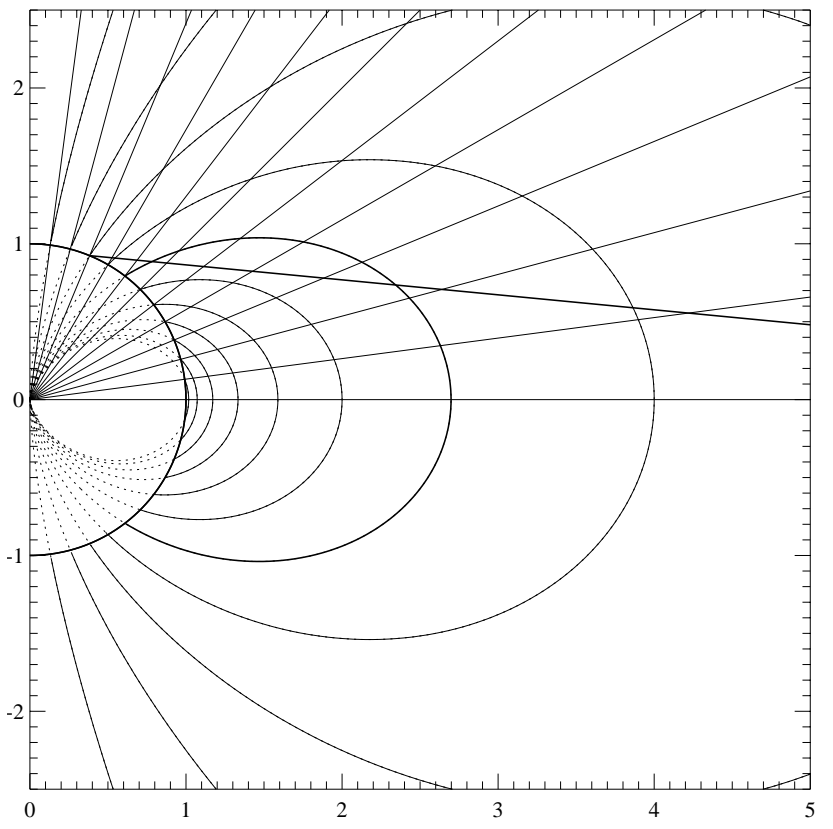

Fig. 1. Relation between ray path, magnetic and geocentric coordinates demonstrated by means of centric dipole coordinates. Straight line rays in a meridian plane. Shells of constant magnetic latitude give dipole field lines in the meridian plane; the surfaces of constant geographic latitudes are concentric cones. There are three possibilities for the relation of the ray to a specific magnetic shell: (a) it cuts the shell twice; (b) it is tangent to the shell; (c) it has no intersection at all if the magnetic latitude is too low. In case (a) the lower intersection point has a larger geocentric latitude than the upper one. The model trough minimum is represented by the magnetic shell which represents its magnetic latitude. Note that invariant magnetic coordinates are calculated with three dimensional field lines and therefore the geometric situation is a bit more complicated.

the model is larger than a few minutes, Fourier or spline interpolation is an adequate method. Gliding third order interpolation might be adequate too and has the advantage that 4 sets of data grids are sufficient at any model time.

\section{The model for the main trough of the $F$ layer}

A complete three dimensional formulation for the main trough has been constructed on the basis of Dynamic Explorer (DE) data (Leitinger and Feichter, 1999)(Feichter and Leitinger, 2002). The model uses the trough minimum model published by Werner and Prölss (1995) (which is also based on DE data) and a time dependent shape of the trough. The shape parameters

- depth of the trough,

- equatorward half-width,

- poleward half-width,

- steepness of the equatorward wall,

- steepness of the poleward wall

have been derived from Dynamic Explorer electron densities gained in the height region below $700 \mathrm{~km}$ and scaled to the

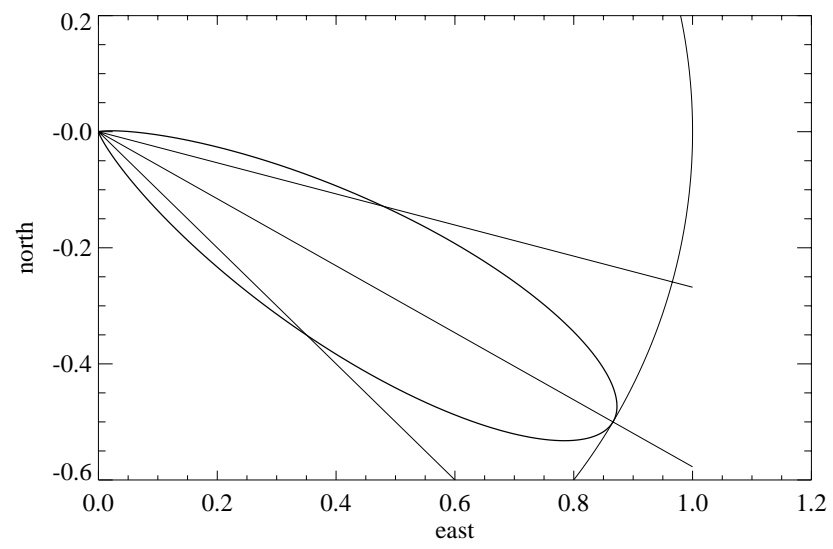

Fig. 2. TID fan beam, projected onto the surface of the earth. Horizontal half width: $30^{\circ}$, azimuth of center: $120^{\circ}$,

F2 layer peak by means of the COSTprof model. Our trough model uses the medians of the shape parameters for three seasons (winter, equinox, summer) and two magnetic local time intervals ("day" and "night")(see Feichter and Leitinger (2002) for statistics). After several tests we settled on a composite for the trough shape. The trough consists of two parts, an equatorward one and a poleward one. Both parts use ellipse sectors for bottom and top joined together by straight lines in such a way that the first derivatives are continuous. The equatorward and poleward parts meet at the trough minimum. Added to the poleward part is a $20 \%$ enhancement which fades out like a Gaussian. For examples of the trough shape see Feichter and Leitinger (2002).

In accordance with the "modulation" requirements the trough model uses height and geographic coordinates as explicit input but depends on

- geomagnetic activity,

- season

- Universal Time.

Internally the trough model depends on magnetic coordinates which are calculated from the geographic coordinates, height, date and time. Since the model for the position of the trough minimum published by Werner and Prölss (1995) uses Invariant Coordinates we have adopted the same type of magnetic coordinates. Magnetic coordinates ensure that the trough features like the walls of the trough are geomagnetic field aligned.

Since magnetic latitude is constant for a given magnetic "shell" all trough features are automatically magnetic field aligned.

\section{The TID model}

The TID model is based on the assumption that Traveling Ionospheric Disturbances are the plasma signatures of Atmospheric Gravity Waves (AGWs). 


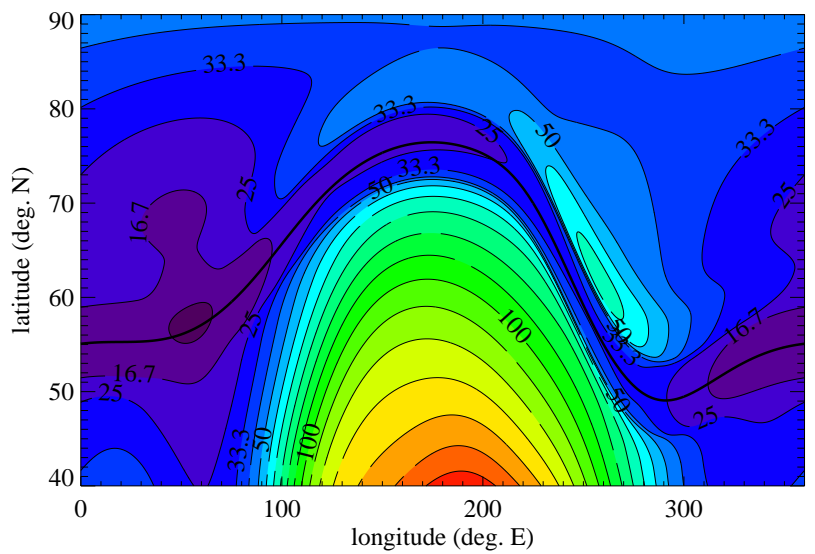

Fig. 3. Example for trough modulation: electron density of the ITU$\mathrm{R}$ (CCIR) "map" for October, $0 \mathrm{UT}, R_{12}=100$ modulated with the main trough for $K_{p}=6$. Isolines of electron density in units of $10^{10} \mathrm{~m}^{-3}$ in a geographic coordinate system.

\subsection{Atmospheric Gravity Waves (AGWs)}

Since we are not dealing with the propagation of AGWs through a realistic atmosphere but need their basic properties only, we can assume validity of the dispersion relation of AGWs in its simplest form (Hines, 1960)

$k_{z}^{2}=\frac{\omega_{b}^{2}-\omega^{2}}{w^{2}} k_{x}^{2}-\frac{\omega_{a}^{2}-\omega^{2}}{c_{s}^{2}}$

$\omega_{b}$ is the (isothermal) Brunt-Väisälä frequency, $\omega_{a}$ the acoustic cut-off frequency, $\omega$ the (angular) frequency of the AGW, $k_{x}$ the horizontal, $k_{z}$ the vertical wave number, $c_{s}$ is the velocity of sound.

$\omega_{b}^{2}=\frac{(\gamma-1) g^{2}}{c_{s}^{2}}, \quad \omega_{a}=\frac{\gamma g}{2 c_{s}}$

Defining horizontal and vertical refractive indices $n_{x}=$ $k_{x} c_{s} / \omega$ and $n_{z}=k_{z} c_{s} / \omega$ and

$a^{2}=\frac{1-\frac{\omega_{a}^{2}}{\omega^{2}}}{1-\frac{\omega_{b}^{2}}{\omega^{2}}}, \quad b^{2}=1-\frac{\omega_{a}^{2}}{\omega^{2}}$,

gives after re-arrangements $\frac{n_{x}^{2}}{a^{2}}+\frac{n_{z}^{2}}{b^{2}}=1$.

This is the equation for a conic section with axes $a^{2}$ and $b^{2}$,

$a^{2}=\frac{\alpha b^{2}}{\alpha-\left(1-b^{2}\right)}$

with $\quad \alpha=\frac{\omega_{a}^{2}}{\omega_{b}^{2}}=\frac{\gamma^{2}}{4(\gamma-1)}>1$.

$(\gamma=1,4 \rightarrow \alpha=1,225)$. The velocity of sound is given by $c_{s}^{2}=\gamma H g \quad(H$ : pressure scale height of the atmosphere, $g$ : acceleration of gravity).

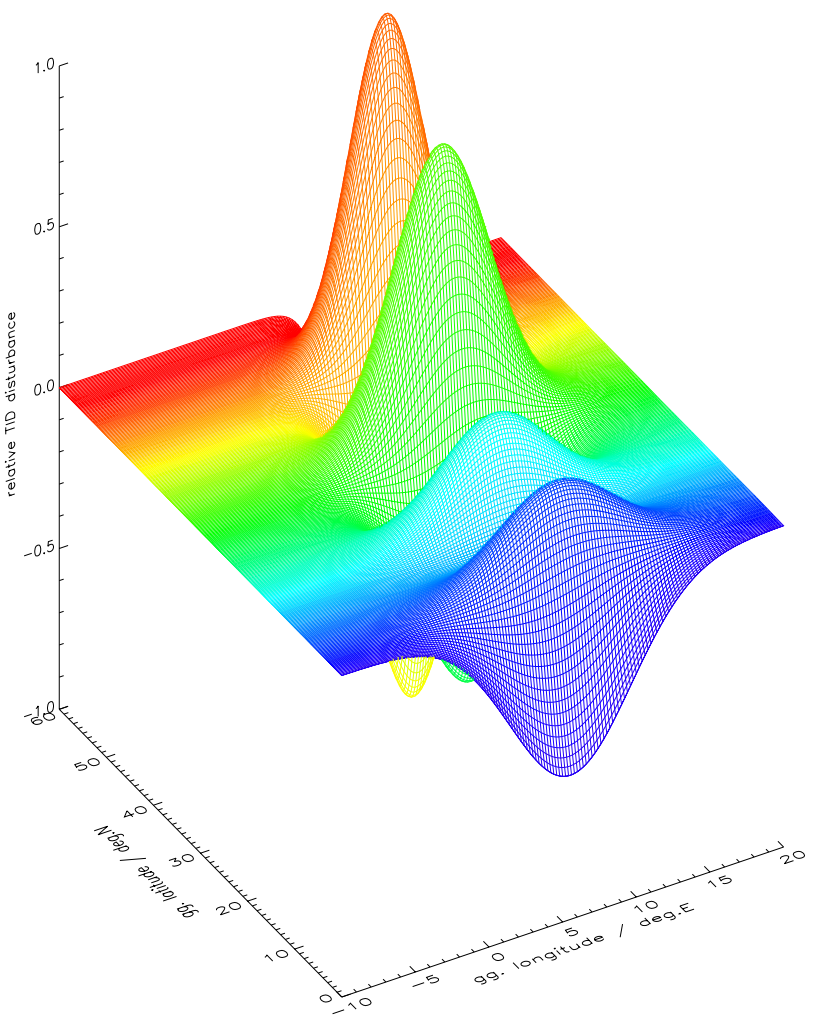

Fig. 4. TID modulation at $250 \mathrm{~km}, 3-\mathrm{D}$ display. Wave period 60 min, hor. wave length $200 \mathrm{~km}$, source point $70^{\circ} \mathrm{N}, 15^{\circ} \mathrm{E}$, fan half width $5^{\circ}$, azimuth $195^{\circ}$.

Real solutions exist in two cases:

1. $b^{2}>0$ (and also $a^{2}>0$ ), meaning $\omega^{2}>\omega_{a}^{2}$. This is the "acoustic branch", the index surfaces are ellipsoids. The refraction indices are always $<1$. For $\omega \rightarrow \infty$ the surfaces degenerate into spheres what corresponds to the propagation of "normal" sound with the phase velocity $c_{S}$. No dispersion and no anisotropy.

2. $b^{2}<0$ and $a^{2}>0$, meaning $\omega^{2}<\omega_{b}^{2}$. This is the branch of "gravity waves" ("buoyancy waves"), the index surfaces are hyperboloids. The refractive indices are always $>1$. For $\omega \rightarrow 0$ the index surfaces degenerate into circular cylinders: $a^{2} \rightarrow \infty, \quad b^{2} \rightarrow$ $-0,225$ (for $\gamma=1,4$ ).

With $c^{2}=-b^{2}$ we have the hyperboloid equation

$\frac{n_{x}^{2}}{a^{2}}-\frac{n_{z}^{2}}{c^{2}}=1, \quad \longrightarrow \quad n_{z}^{2}=c^{2}\left(\frac{n_{x}^{2}}{a^{2}}-1\right)$.

A (gravity branch) AGW can be defined by $\omega<\omega_{b}$ (or $\tau>\tau_{b}$ ) and $k_{z} . \quad k_{z}$ and $\omega$ give $n_{x}, a, c$ and finally $n_{z}$, $k_{z}=n_{z} \omega / c_{s}, \lambda_{z}=2 \pi / k_{z}$. 


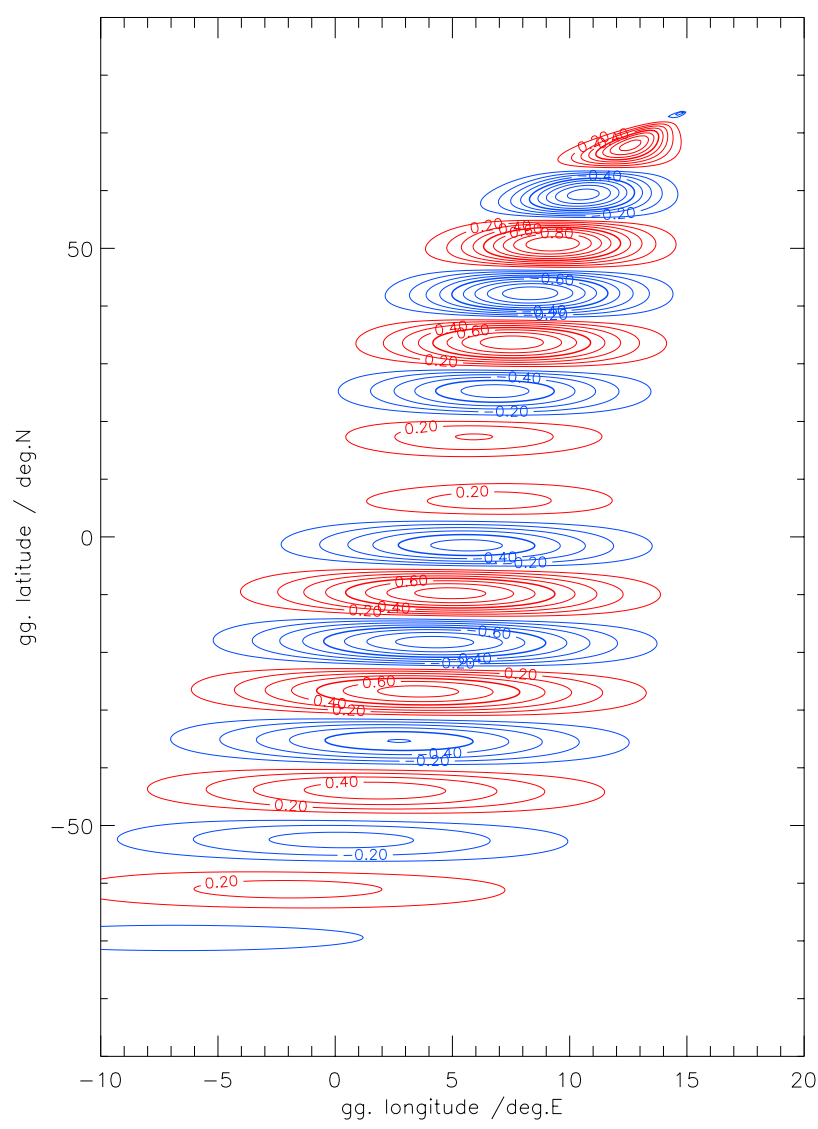

Fig. 5. TID modulation at $250 \mathrm{~km}$, contour line display. TID properties as for Fig. 4. Red: positive values, blue: negative values.

\subsection{TIDs as plasma signatures of AGWs}

To find the plasma signature of an AGW we need the equation of continuity for the electron density

$\frac{\partial N_{e}}{\partial t}=q-L-\nabla \cdot\left(N_{e} \boldsymbol{u}_{e}\right)$

$N_{e}$ : electron density; $u_{e}$ : electron velocity. Perturbation ansatz (compare, e.g., Leitinger (1992)): $N_{e}=N_{e o}+$ $N_{e 1}, \quad \boldsymbol{u}_{e}=\boldsymbol{u}_{e o}+\boldsymbol{u}_{e 1}$. Index $o$ : background, index 1: perturbation (induced by AGW).

Neglecting a background electron "wind" (setting $\boldsymbol{u}_{e 0}=$ 0 ) and assuming that the passing AGW influences the electrons only by the transport term (no effect on production and loss) gives

$\frac{\partial N_{e}}{\partial t}=-\nabla \cdot\left(N_{e o} \boldsymbol{u}_{e 1}\right)$

The relation of $\boldsymbol{u}_{e 1}$ to $\boldsymbol{u}_{n}$ is given by a balance between the Lorentz force and the "ion drag" force:

$e\left(\boldsymbol{u}_{e 1} \times \boldsymbol{B}\right)+m_{e} v_{e n}\left(\boldsymbol{u}_{e 1}-\boldsymbol{u}_{n}\right)=0$

$\left(\boldsymbol{B}\right.$ : geomagnetic induction vector; $e$ : electron charge; $m_{e}$ : electron mass; $v_{e n}$ : effective collision frequency).
Introducing the unit vector of the geomagnetic field $\boldsymbol{b}$ by $\boldsymbol{B}=B \boldsymbol{b}$ and the electron gyro frequency $\omega_{g}=\left(e / m_{e}\right) B$ gives the solution

$\boldsymbol{u}_{e 1}=\left(1+\alpha^{2}\right)^{-1}\left(\alpha^{2} \boldsymbol{u}_{n}-\alpha \boldsymbol{u}_{n} \times \boldsymbol{b}+\left(\boldsymbol{u}_{n} \cdot \boldsymbol{b}\right) \boldsymbol{b}\right)$

with $\quad \alpha=\frac{v_{e n}}{\omega_{g}}$

In the F region $v_{e n} \ll \omega_{g}$ which justifies the approximation $\boldsymbol{u}_{e 1} \doteq\left(\boldsymbol{u}_{n} \cdot \boldsymbol{b}\right) \boldsymbol{b}$.

A monochromatic wave in $\boldsymbol{u}_{n}$ gives a monochromatic wave in $\boldsymbol{u}_{e 1}$ and in $N_{e 1}$. Using a relevant ansatz allows to replace $\partial A / \partial t$ by $j \omega A$ and $\nabla A$ by $j \boldsymbol{k} A(j=\sqrt{-1})$.

$j \omega N_{e 1}=\left(\boldsymbol{u}_{n} \cdot \boldsymbol{b}\right)\left[j(\boldsymbol{k} \cdot \boldsymbol{b}) N_{e 0}-(\boldsymbol{b} \cdot \nabla) N_{e o}\right]$

$N_{e 1}=\frac{\left(\boldsymbol{u}_{n} \cdot \boldsymbol{b}\right)}{\omega}[(\boldsymbol{k} \cdot \boldsymbol{b})-j(\boldsymbol{b} \cdot \nabla)] N_{e o}$

If the second term in [ ] can be neglected we approximate

$\frac{N_{e 1}}{N_{e o}} \doteq \frac{k\left(\boldsymbol{u}_{n} \cdot \boldsymbol{b}\right)}{\omega} \cos (\Xi)$

Since we have a real and an imaginary part of $\boldsymbol{u}_{n}=\boldsymbol{u}_{r}+j \boldsymbol{u}_{i}$ $\left(\boldsymbol{u}_{n} \cdot \boldsymbol{b}\right)=\left(\boldsymbol{u}_{r} \cdot \boldsymbol{b}\right)+j\left(\boldsymbol{u}_{i} \cdot \boldsymbol{b}\right)$

Using local Cartesian coordinates $(x, y, z)$

$\boldsymbol{k}=\left(\begin{array}{l}k_{x} \\ k_{y} \\ k_{z}\end{array}\right), \boldsymbol{u}_{\boldsymbol{r}}=\left(\begin{array}{l}u_{r x} \\ u_{r y} \\ u_{r z}\end{array}\right), \boldsymbol{u}_{i}=\left(\begin{array}{l}u_{i x} \\ u_{i y} \\ u_{i z}\end{array}\right), \boldsymbol{b}=\left(\begin{array}{l}b_{x} \\ b_{y} \\ b_{z}\end{array}\right)$ gives $\cos (\Xi)=k_{x} b_{x}+k_{y} b_{y}+k_{z} b_{z}$,

$$
\begin{aligned}
\left(\boldsymbol{u}_{n} \cdot \boldsymbol{b}\right)= & \left(u_{r x} b_{x}+u_{r y} b_{y}+u_{z} b_{r z}\right)+ \\
& j\left(u_{r x} b_{x}+u_{r y} b_{y}+u_{r z} b_{z}\right)=\left|\left(\boldsymbol{u}_{n} \cdot \boldsymbol{b}\right)\right| \exp (j \Psi)
\end{aligned}
$$

$\frac{N_{e 1}}{N_{e o}} \doteq\left|\frac{\left(\boldsymbol{u}_{n} \cdot \boldsymbol{b}\right)}{v_{p h}}\right| \cos (\Xi) \cos \left[k_{x} s+k_{z} h+\Psi(h)-\omega t\right]$

The phase constant $\Psi$ reflects the polarization of the AGW: According to Beer (1974), the ratio of horizontal to vertical AGW wind velocity components is

$\frac{\left\{c_{s}^{2}\left[k_{z}+j /(2 H)\right]-j g\right\} k_{x}}{\omega^{2}-c_{s}^{2} k_{x}^{2}}$ leading to

$\tan \Phi=\frac{\gamma-2}{2 \gamma k_{x} H}$

Since we have assumed height constant $\gamma, k_{x}, H$ the phase constant of the AGW, $\Phi$, but not that of the TID, $\Psi$, is independent of height.

The amplitude of $\boldsymbol{u}_{n}$ is also height dependent: (a) We have to take into account the exponential increase of amplitudes 

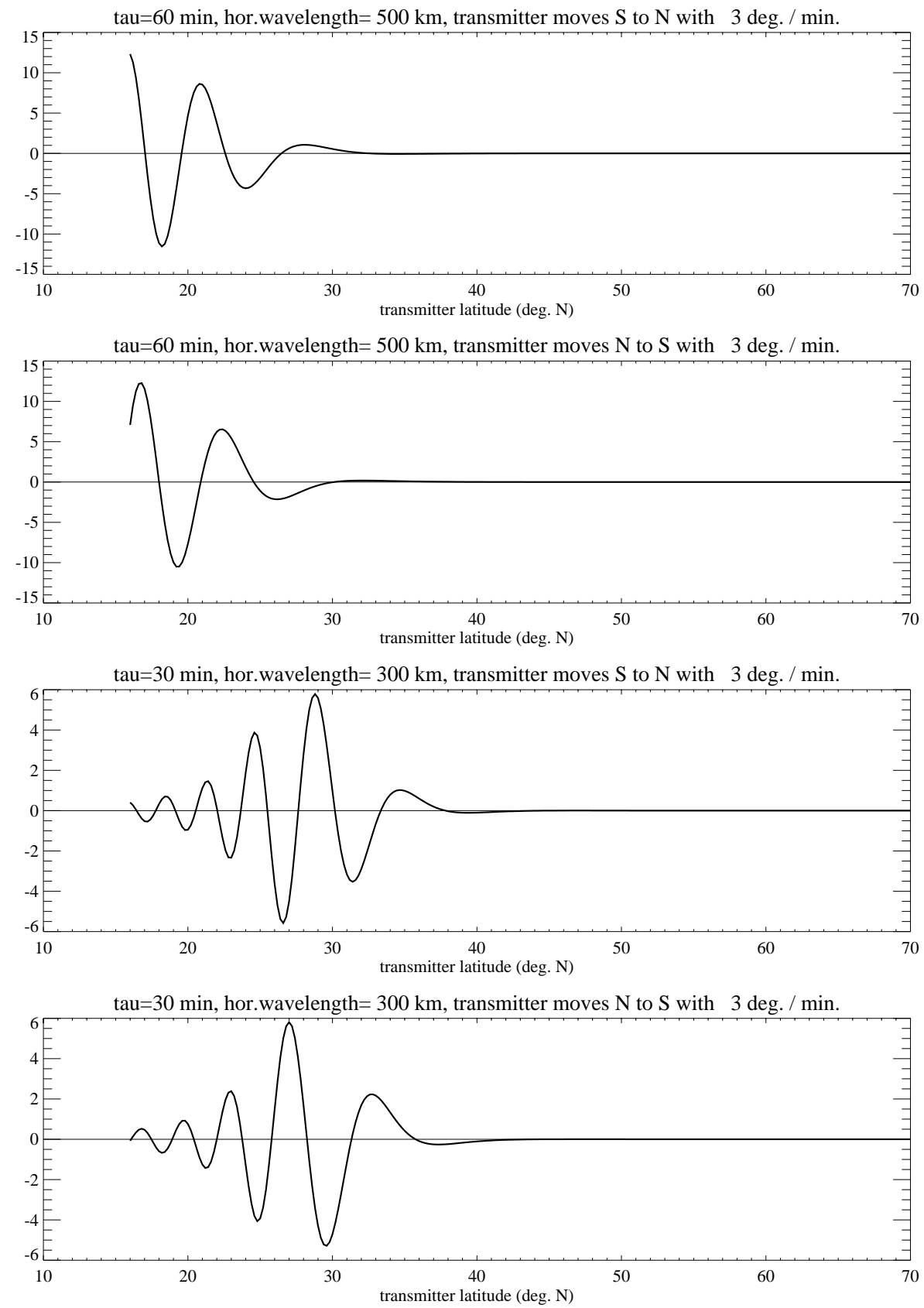

Fig. 6. TID modulation in slant electron content for a ground station at $45^{\circ} \mathrm{N}, 15^{\circ} \mathrm{E}$ and a (satellite) transmitter moving at $1000 \mathrm{~km}$ with 3 degrees per minute in the meridian plane of the ground station. TID properties: Source point at $70^{\circ} \mathrm{N}, 30^{\circ} \mathrm{E}$, fan beam with half width of $10^{\circ}$, azimuth of center: $190^{\circ}$. With panel numbers 1 for top, 4 for bottom: wave periods 60 minutes (panels 1 and 2 ) and 30 min (panels 3 and 4); horizontal wavelengths $500 \mathrm{~km}$ (panels 1 and 2) and $300 \mathrm{~km}$ (panels 3 and 4); transmitter at a height of $1000 \mathrm{~km}$ moves S to N (panels 1 and 3 ) or $\mathrm{N}$ to $\mathrm{S}$ (panels 2 and 4).

with $\exp \left[\left(h-h_{o}\right) /(2 H)\right]$ (needed to compensate for the exponential decrease of neutral atmosphere density) and damping through ion drag. Since there is indication that the relative amplitude of LSTIDs peaks below the F layer peak we have chosen a Chapman layer type height dependence:

$\frac{\left|U_{n}\right|}{v_{p h}}=\left(\frac{\left|U_{n}\right|}{v_{p h}}\right)_{0} \exp [1-z-\exp (-z)]$ with $z=\frac{h-h_{o}}{H_{C h}}$

$h_{o}$ being the height of the amplitude maximum, $H_{C h}$ a thickness parameter.

Since we know that the AGWs with sources in the auroral zone are not radiated isotropically but according to a distinct horizontal radiation pattern, we had to assume such a pattern and have adopted radiation into beams using the for- 

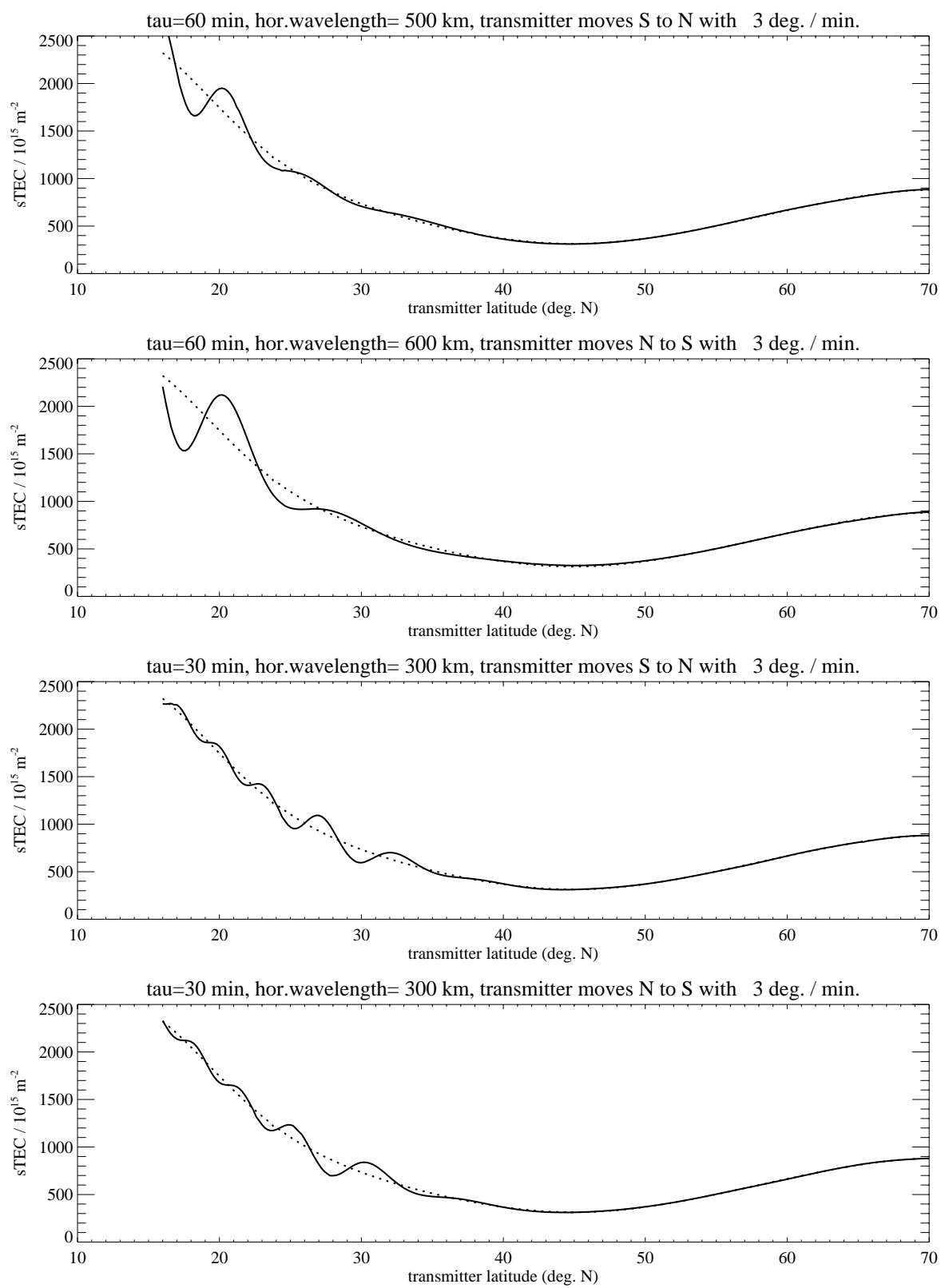

Fig. 7. NeQuick for October, 12:00 UT, solar activity parameter $\mathrm{R}_{12}=150$ modulated with the TID disturbances used for Fig. 6. Slant electron content in units of $10^{15} \mathrm{~m}^{-2}$ vs. geogr. latitude of the transmitter.

mula $A(\sigma)=A\left(\sigma_{o}\right) \cos ^{\kappa}\left[\left(\sigma-\sigma_{o}\right) / 2\right], \sigma$ being the azimuth of a ray emitted from the source point, $\sigma_{o}$ being the azimuth in which the maximum amplitude is emitted. Small beams need large values of $\kappa$ : A half width of $\delta$ degrees needs approximately $\kappa \doteq 18200 / \delta^{2}$ (valid if $\delta<10$ degrees), e.g., $\delta=2$ degrees needs $\kappa=4550$.

It is also appropriate to include geometric dilution of the TIDs. Distribution of wave energy over a sphere leads to a dilution of the amplitudes proportional to $1 / \sqrt{\sin \Psi}$. To avoid problems with the source point $(\Psi=0)$ we use $1 / \sqrt{1+\left(\sin \Psi / \sin \Psi_{o}\right)}$ instead.

Finally, ion drag extracts energy from the AGW which means that we have to take attenuation into account. We have chosen attenuation according to $\exp \left(-\Psi / \Psi^{*}\right)$ with $\Psi^{*}=\pi \lambda_{s} / R_{e}$.

The simplifying assumptions are sufficiently good for the "far field" in the F region but should not be applied in the vicinity of the source region of the AGWs and not in the $\mathrm{E}$ region.

\section{3 (MS and LS) TID modulation}

The TID model adopted:

- The TIDs are assumed to be plasma signatures of Atmospheric Gravity Waves (AGWs)

- The TIDs originate in chosen sources points 
- a TID travels in a "fan beam" defined by its azimuth and its half width

- the wave properties are given by the wave period $\tau$ and by the horizontal wave length $\lambda_{h}$

- for the vertical structure a Chapman profile was adopted defined by a scale height $H_{T}$ and by a peak height $h_{m T}$. Values chosen for the examples shown: $H_{T}=100 \mathrm{~km}$, $h_{m T}=250 \mathrm{~km}$

- the forward tilt of the wave fronts is produced via a height dependent phase constant $\Psi$ in accordance with the dispersion relation of the AGWs

- the geometric dilution of horizontally traveling AGWs and horizontal attenuation are also taken into account

- the model allows superposition of several TID wave trains.

The following properties of the AGW are needed.

- Horizontal component $k_{x}$ and vertical component $k_{z}$ of the wave vector $\boldsymbol{k}$

- AGW period $\tau=2 \pi / \omega$

- Velocity of the disturbance $\boldsymbol{u}_{n}=\boldsymbol{U}_{n} \cos \left[k_{x} s+k_{z} h+\right.$ $\Phi(h)-\omega t]$ ( $s$ : horizontal coordinate, $h$ : height, $t$ : time, $\Psi$ is a height dependent phase constant.

Derived quantities:

$\lambda_{x}=2 \pi / k_{x} ; \lambda_{z}=2 \pi / k_{z}$ : horizontal and vertical wave lengths;

$\lambda=2 \pi / k:$ (total) wave length $\left(k=|\boldsymbol{k}|=\sqrt{k_{x}^{2}+k_{z}^{2}}\right) ;$

$\omega / k=\omega / \sqrt{k_{x}^{2}+k_{z}^{2}}=v_{p h}$ : phase velocity (to be distinguished from $v_{h}=\omega / k_{x}$ the horizontal phase velocity.

\section{Modulation examples}

A few examples for model modulation are shown here: Fig. 3 shows an F2 layer peak density map modulated with the main trough. For more examples of trough modulations see Leitinger et al. (2002).

Figures 4 and 5 are two different displays of a large scale TID modulation in electron density at $250 \mathrm{~km}$ height (peak of the TID amplitude). An example for the TID modulation in electron content is shown in Fig. 6. Modulated electron content is displayed in Fig. 7.

\section{Conclusions}

The method to "modulate" electron density models by multiplication is very versatile. Since the sub-models are time dependent, we are able to provide realistic "scenarios" for assessment and case studies. We can introduce highly dynamic structures for which the wavelike TID disturbances are only one example. More dynamic structures are in construction, e.g., equatorial "bubbles", high latitude "blobs" and "patches" and soliton like disturbances to imitate some of the observations made during ionospheric storms.

\section{References}

Beer, T.: Atmospheric waves, Wiley, New York and Toronto, 1974.

Bilitza, D.: International Reference Ionosphere 2000, Radio Sci., 36, 261-275, 2001.

Feichter, E. and Leitinger, R.: Properties of the main trough of the F region derived from Dynamic Explorer 2 data, Ann. Geophysics, 45, 117-124, 2002.

Hines, C. O.: Internal atmospheric gravity waves at ionospheric heights, Canad. J. Phys., 38, 1441-1481, 1960.

Leitinger, R.: Travelling Ionospheric Disturbances (TIDs): Wissensstand und neuere Entwicklungen, Kleinheubacher Ber., 35, 1-14, 1992.

Leitinger, R. and Feichter, E.: Modelle für den Trog der F Schicht: Möglichkeiten und Grenzen, Kleinheubacher Ber., 42, 63-70, 1999.

Leitinger, R., Hochegger, G., Hafner, J., Radicella, S., and Nava, B.: NeQuick, COSTprof, NeUoG-plas - eine Familie von Ionosphärenmodellen, Kleinheubacher Ber., 43, 20-25, 2000.

Leitinger, R., Nava, B., Hochegger, G., and Radicella, S.: Ionospheric profilers using data grids, Phys. Chem. Earth(C), 26, 293-301, 2001.

Leitinger, R., Radicella, S., and Nava, B.: Electron density models for assessment studies - new developments, Acta Geodet. Geophys. Hung., 37, 183-193, 2002.

Werner, S. and Prölss, G. W.: The position of the ionospheric trough - an empirical model based on DE2 observations, Kleinheubacher Ber., 38, 143-154, 1995. 